\title{
Determinación de residuos de líquidos inflamables en escombros de incendios por cromatografía de gases-FID
}

\author{
Henry Daniel Ponce Rodríguez*
}

\section{RESUMEN}

Se presenta un método para determinar trazas de líquidos inflamables en escombros de incendios, tales como gasolina, diesel y otros compuestos acelerantes, por medio de cromatografía de gases con detector de ionización de flama (FID), capaz de separar e identificar en cantidades de partes por millón con buena resolución y exactitud. Se prepararon muestras control para corroborar el proceso de extracción por medio de la técnica de adsorción pasiva con tiras de carbón activado y su posterior desorción con disulfuro de carbono ( $\mathrm{CS}_{2}$ ), utilizando una columna capilar de 30 metros DB-1, con una corrida cromatográfica de 31 minutos. Se obtuvo una correcta separación capaz de diferenciar entre compuestos más volátiles de aquellos con elevado punto de ebullición. Se presentan los cromatogramas obtenidos de los controles blanco, acelerantes y disulfuro; todos preparados asemejando las posibles matrices a estudiar en un posible incendio, tales como restos de papel, ropa, algodón, entre otros más.

Palabras clave: Líquidos inflamables, Cromatografía de gases, Detector FID, Adsorción pasiva, Muestras Control, Matriz, Cromatograma.

We present a method for determining traces of flammable liquids in fire debris, such as gasoline, diesel and other compounds accelerators, by gas chromatography with flame ionization detector (FID) capable of separating and identifying in quantities of parts per million with good resolution and accuracy. Control samples were prepared to verify the extraction process by passive adsorption technique with strips of activated coal and subsequent desorption with carbon disulfide $\left(\mathrm{CS}_{2}\right)$ using a capillary column $30 \mathrm{~m} \mathrm{DB}-1$, with a chromatographic run of 31 minutes. Was obtained a correct separation able to differentiate between compounds more volatile than those with high boiling point. Presents the chromatogram obtained from blank controls, and disulfide accelerators, all possible matrix resembling prepared to study a possible fire, such as waste paper, cloth, cotton, among others.

Key words: Flammable liquids, gas chromatography, detector FID, passive adsorption, Control Samples, Matrix, Chromatogram.

*Universidad Nacional Autónoma de Honduras. Facultad de Química y Farmacia. Toxicólogo Forense Laboratorio Químico-Toxicológico, Dirección de Medicina Forense. henryponcer@gmail.com 


\section{INTRODUCCIÓN}

El desarrollo y aplicación de la Química abarca diferentes campos, tales como la industria, la salud, tecnología y la educación, entre muchos otros. Uno de esos campos es el de la Química Forense, la cual trata de dar las bases científicas en el esclarecimiento de delitos, a fin de que impere la verdad a través de la evidencia técnica científica.

Junto a los desastres naturales, los incendios representan las mayores pérdidas de la propiedad y la vida humana en todo el mundo. Un incendio intencional, la creación deliberada de fuego para destruir la propiedad o para tomar la vida humana, es uno de los crímenes más difíciles de investigar, ya que mucha de la evidencia en el lugar de los hechos es destruida por el fuego o en el proceso de extinción del mismo.

La cromatografía de gases es uno de los métodos analíticos más utilizados, en diversos campos, debido a sus múltiples ventajas en la separación y determinación de compuestos volátiles mediante la partición del analito entre una fase gaseosa móvil y una fase estacionaria recubierta. Un gráfico que se produce, llamado Cromatograma, representa el tiempo de retención de cada compuesto (la cantidad de tiempo del compuesto retenido en la columna) frente a la respuesta del detector en términos de abundancia.

El cromatógrafo de gases se compone en general por seis partes: un gas portador, generalmente nitrógeno o helio; un puerto de inyección, a cierto grado de temperatura capaz de volatilizar la muestra; un horno, el cual es responsable de proporcionar las rampas de temperatura idóneas para la correcta separación de los componentes de la muestra; una columna capilar de carácter no polar que permita la separación en base a los puntos de ebullición de los componentes; el detector, en el presente estudio se trabajó con el detector de ionización de flama (FID), que trabaja por la combustión de eluyente de la columna en una llama para producir una corriente de iones, dicha corriente resultante es amplificada, medida y registrada; por último, un dispositivo de grabación de datos, en ese aspecto la interpretación de los datos no se basa en la concentración de un compuesto u otro, sino más bien en la respuesta del detector a un compuesto.

El método es capaz de identificar líquidos inflamables a base de petróleo, los cuales representan el mayor número de productos utilizados como acelerantes de incendios, y que suelen ser estos productos los de interés en el análisis de restos de incendios, principalmente aquellos en el rango de n-pentano al eicosano, con 
puntos de ebullición entre los 36 y 205 C. Las clases comunes en este rango son la gasolina, destilados de petróleo, productos iso-parafínicos, nafténicos, disolventes aromáticos y los productos normales de alcano.

El estudio busca, además, comprobar la eficacia del proceso de extracción por medio de la adsorción pasiva con carbón activado, utilizando tiras de carbón activado, en un recipiente cerrado herméticamente exponiendo a una temperatura entre 80 y $90 \mathrm{C}$ durante al menos 17 horas, con la consecuente desorción haciendo uso del disulfuro de carbono, finalizando con el análisis instrumental anteriormente detallado.

En última instancia se interpretan los datos obtenidos realizando las consideraciones que el estudio de las muestras amerita, mismas que son mínimas en los controles preparados en el presente, pero de vital importancia en los casos prácticos, así como detallar algunos de los escenarios posibles para explicar los hallazgos del laboratorio.

La idea principal es desarrollar un método capaz de determinar la presencia de dichas sustancias, con un buen límite de detección, rápido, confiable y de acorde a los insumos con que cuenta el Laboratorio Químico-Toxicológico, de la Dirección de Medicina Forense, que pueda ofrecer una prueba científica válida y verdadera en los procesos jurídicos relacionados con el uso de líquidos inflamables con el fin de provocar un incendio.

\section{MÉTODOSYTÉCNICAS}

\section{Diseño}

Se trabaja en primera instancia con gasolina y diesel comerciales, analizándolos en el cromatógrafo de gases para observar el comportamiento de las sustancias como tal, además de corroborar el desempeño del equipo con el método propuesto. Además en el estudio se prepararon controles positivos de gasolina y diesel, así como un control negativo. Todos ellos consistían en colocar en latas de metal sin revestimiento con cierre hermético, pedazos de materiales adsorbentes de líquidos inflamables, tales como ropa, papel y algodón, entre otros.

\section{Población}

Idealmente se pensó trabajar con estándares certificados de gasolina y de diesel, pero por falta de recursos de la institución, así como debido a la conveniencia de 
trabajar con los productos comerciales, ya que serán estos mismos los que se pueden encontrar en un futuro caso de incendio.

\section{Entorno}

Se realizaron todas las pruebas en el Laboratorio Químico-Toxicológico, de la Dirección de Medicina Forense, laboratorio encargado de diferentes análisis en el campo de la química forense, así como del tipo toxicológico.

\section{Intervenciones}

La técnica de extracción fue la de adsorción pasiva con tiras de carbón activado, utilizando un horno de convección, marca Precisión, modelo economy oven, en una temperatura de trabajo de $80 \mathrm{C}$. Se utilizó una cromatografía de gases marca Varian, con una columna capilar DB-1, utilizando nitrógeno ultra puro (UHP) como gas de acarreo, con una temperatura de inyección de 230C, un detector FID a 250C utilizando hidrógeno ultrapuro y el programa de la compañía Varian workstation.

\section{Estadística}

Dado la naturaleza del estudio, en donde lo que se busca es desarrollar un método para determinar la presencia o no de los líquidos inflamables, no se ahonda sobre cálculos estadísticos, pero sí se analizan los diferentes cromatogramas obtenidos, centrando la mayor atención en los picos característicos de la gasolina y el diesel, utilizando como apoyo la literatura sobre derivados del petróleo.

\section{CAPÍTULO I}

\section{Muestreo}

Pese a que el presente trabajo se refiere al desarrollo de un método para la identificación de los compuestos acelerantes, y que en la mayor parte de los casos la toma de muestras es responsabilidad de otros organismos de investigación, quedando delimitada la labor del laboratorio en el desarrollo de los análisis y futura interpretación de los datos recolectados del análisis; es preciso referirnos brevemente al tema de muestreo, en lo que respecta a la adquisición, manejo, manipulación y almacenamiento de los residuos de escombros, pero debido a la poca experiencia con que cuentan los entes investigativos, es en general el laboratorio quien presenta las directrices, observaciones y recomendaciones sobre dicho proceso. 
En general encontramos ciertas recomendaciones al momento de la selección de la muestra, mismos que nos servirán de guía en nuestro trabajo, para poder desarrollar un método de análisis que valore el efecto de la matriz en los resultados obtenidos, volviendo los resultados más confiables.

Entre dichas recomendaciones mencionaremos las siguientes:

1) La toma de muestras deberá realizarse tan pronto como sea posible, después que el fuego haya sido controlado. Debido a que existen procesos de evaporación, dilución o dispersión que disminuyen las oportunidades de colectar muestras positivas en caso de existir la presencia de líquidos inflamables.

2) El mejor lugar para recolectar muestras es en la periferia de los llamados "patrones de fluidez", lo que se evidencia a menudo por la interfaz distinta entre las zonas más frecuentemente dañadas y las zonas menos dañadas.

3) Materiales absorbentes como alfombras, madera blanda, tejido de la ropa, ropa de cama o cortinas, papel y el suelo, son mejores a tomar que aquellos matinales no absorbentes como el vidrio, baldosas y concreto.

El embalaje de la evidencia es de suma importancia para la preservación y protección de la evidencia, entre el tiempo en que se recolecta la misma y el momento en que esta será analizada en el laboratorio.

Debido a la naturaleza volátil de los líquidos inflamables, las muestras de escombros deben colocarse en contenedores herméticos, siendo el mejor contenedor para residuos de líquidos inflamables los de latas de metal de tipo de pintura, pero sin recubrimiento, con tapas herméticas a presión.

Tomando en cuenta lo expuesto anteriormente, se prepararon controles positivos y negativos en latas metálicas de capacidad de un galón, cerradas herméticamente (figura 1.1).

Se introdujo en cada uno residuos de materiales como papel, tela, algodón, todos materiales con buena absorción, en iguales cantidades, evitando una sobresaturación de los mismos. 


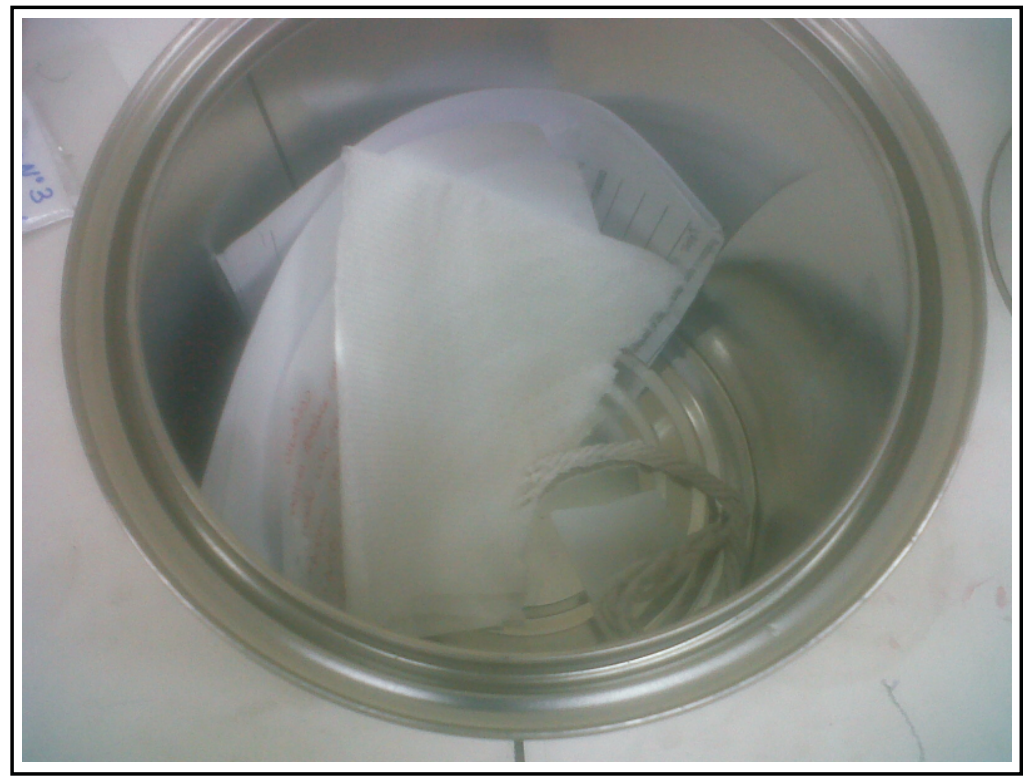

Figura 1.1. Preparación de los controles de gasolina y diesel, con materiales absorbentes.

En el caso de los controles positivos de gasolina y diesel, se procedió a adicionar la cantidad de 1000 microlitros de cada especie en el recipiente correspondiente, y para el caso del control de matriz, este fue preparado adicionando únicamente los materiales absorbentes, lo que en los resultados servirá para observar los posibles efectos de matriz, valorando la significancia de la misma, así como en el descarte de las señales encontradas en el cromatograma, que puedan hallarse en los controles, lo que se traduce en la especificidad del método. (Figura 1.2)

\section{CAPÍTULO॥}

\section{Técnica de extracción y preparación}

Las técnicas de separación de muestras más utilizadas son el proceso de adsorción. Adsorción es la concentración de gas o moléculas de líquido en la superficie de un sólido, llamado apropiadamente un adsorbente. En el análisis de escombros el proceso de adsorción es exclusivo en el sistema de "headspace", aunque ciertos analitos pueden separarse directamente de muestras líquidas de manera satisfactoria.

En la aplicación de headspace, el adsorbente es introducido en el espacio que 
queda entre la tapa y la muestra, dentro del contenedor, y todos los compuestos volátiles son colectados y concentrados. El adsorbente es removido posteriormente y las especies adsorbidas se someten a una desorción para ser analizadas. (Figura 2.1).

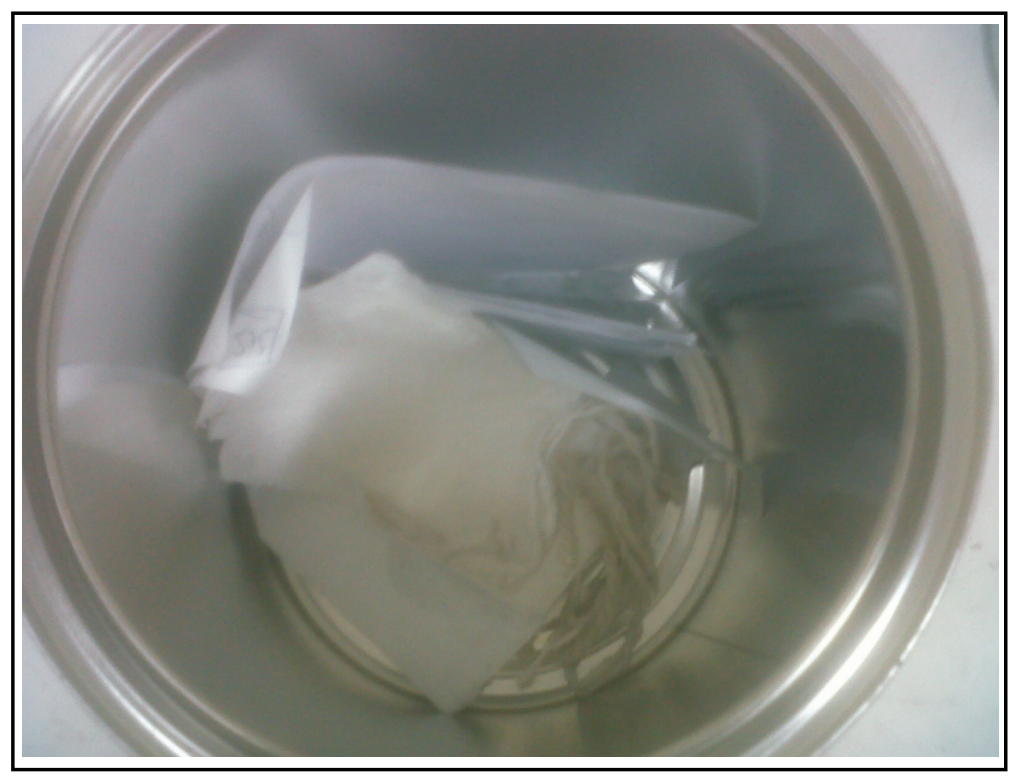

Figura 1.2. Preparación del control de matriz, con materiales absorbentes.

Primero y ante todo, el adsorbente seleccionado debe favorecer compuestos no polares. El carbón encendido, también llamado carbón activado, es el adsorbente utilizado en el análisis de los restos de fuego y generalmente es producido por la destilación destructiva de conchas de coco. Siendo especialmente utilizado para la adsorción de compuestos hidrocarburos no polares, específicamente para la colección de compuestos con un amplio rango de puntos de ebullición ( 0-260C). La eficacia de la adsorción de carbono disminuye al aumentar la polaridad, por lo tanto más compuestos polares (generalmente producidos desde la matriz) presentes en el headspace se adsorberán en menor cantidad.

Adicionalmente, con el carbón activado, compuestos aromáticos poseen mucha mayor afinidad que los compuestos alifáticos. Típicamente, a menos que el absorbente se encuentre sumamente saturado, es poco probable que dicha preferencia afectara a la medida de recuperación para la correcta identificación de líquidos inflamables presentes, siempre y cuando el analista esté consciente y compense este fenómeno en la fase de interpretación de datos. 
El carbón activado es especialmente adecuado para el muestreo de hidrocarbonos entre $\mathrm{C} 5$ y $\mathrm{C} 18$, y realizado adecuadamente para los compuestos $\mathrm{C} 18-\mathrm{C} 20$, al estar estos adecuadamente representados en el sistema de "headspace". El volumen adecuado para el "headspace" debe ser tal que haya suficiente espacio entre los compuestos volátiles a difundirse. Un volumen insuficiente favorece generalmente a los compuestos de bajo punto de ebullición, lo que puede resultar en un cambio significativo en el aparente intervalo de ebullición de los líquidos inflamables. Este es un problema típico cuando se utilizan contenedores no rígidos (bolsas de polímeros) en la recolección de las muestras.

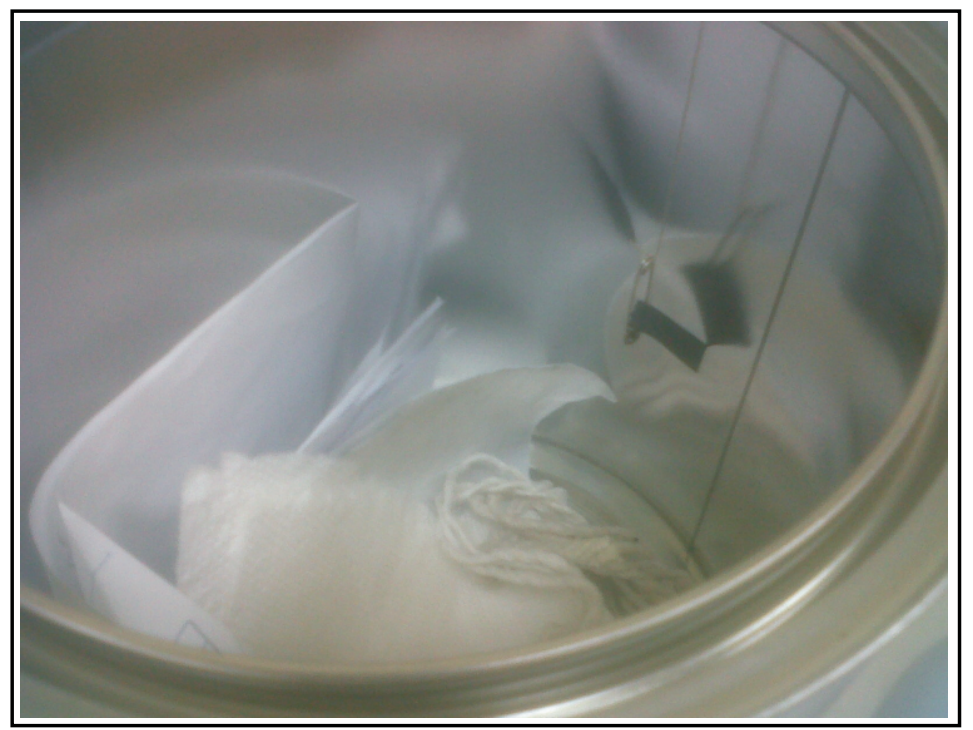

Figura 2.1. Desarrollo del Headspace para los controles.

La composición de la matriz es otro factor significante en el proceso de adsorción de los líquidos inflamables de los restos de incendios. Materiales absorbentes y adsorbentes en la matriz de la muestra inhibirán la volatilización. Restos carbonizados, si bien no son tan eficientes en las propiedades adsortivas como en las tiras de carbón activado, generalmente retienen compuestos de alto peso molecular y en algunos casos compuestos aromáticos, por lo que esos tipos de compuestos serán poco representados en el headspace, y por lo tanto no se encontrarán al momento de la etapa de análisis de la muestra. En general, en la presencia de restos carbonizados, la adsorción de compuestos que eluyen por encima de $\mathrm{C} 18$ no serán consistente o proporcionadamente representados. Existes excepciones cuando la concentración de líquidos inflamables es muy elevada o las propiedades de adsorción de la matriz no son tan grandes. 
Finalmente, el factor más determinante en el sistema de extracción es el de la temperatura. La temperatura de adsorción impacta en la composición final de las especies adsorbidas de dos maneras diferentes: temperaturas elevadas favorecen la volatilización de los compuestos que mejor representan los intervalos de ebullición de los líquidos inflamables a base de petróleo; bajas temperaturas reducirán la velocidad de desplazamiento, lo que puede distorsionar la concentración relativa de los componentes individuales de la especie adsorbida. Temperaturas elevadas se traducirá en la desorción preferencial de compuestos con puntos de ebullición inferiores, cambiando así la composición de la especie adsorbida final a los compuestos de punto de ebullición superiores. Las técnicas de "headspace" de adsorción generalmente usan las temperaturas en el rango de 60$80{ }^{\circ} \mathrm{C}$ para evitar estos dos efectos. Temperaturas en ese rango se consideran ideales para la búsqueda general y muestreo de las diferentes clases de líquidos inflamables. (Figura 2.2).

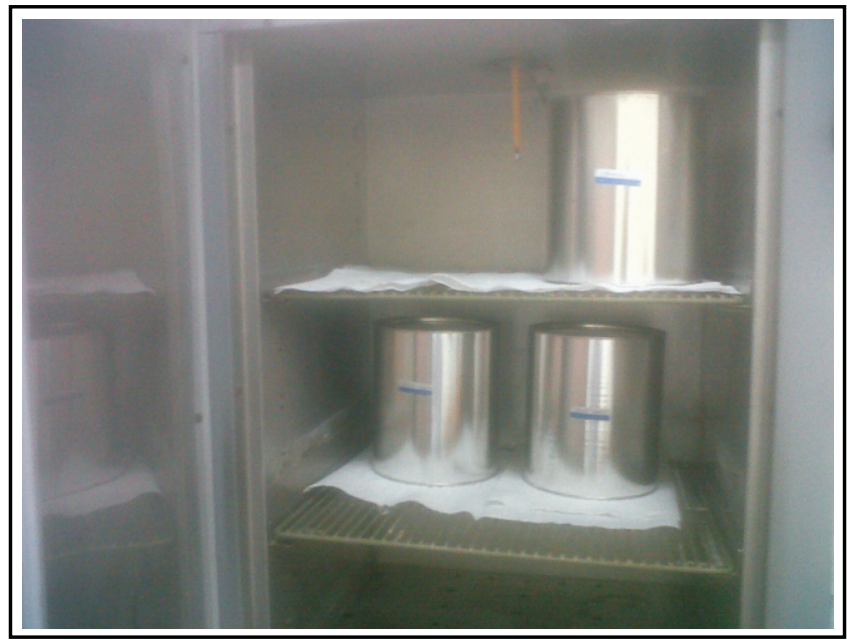

Figura 2.2. Colocación de los controles en el horno.

\section{CAPÍTULO III}

\section{Análisis Instrumental}

La cromatografía incluye una serie de técnicas que tienen en común la separación de las especies como resultado de la partición, por una desorción diferencial, entre dos fases diferentes, una estacionaria con una larga superficie de contacto y la otra, una fase móvil en contacto con la primera (Figura 3.1). La cromatografía no está restringida a las separaciones analíticas, puede también ser utilizada en la 
separación de sustancias puras, el estudio de cinética de reacciones, investigaciones a nivel de estructura molecular, y la determinación de constantes fisicoquímicas, incluyendo constantes de estabilidad de complejos, entalpia, entropía y energía libre. La cromatografía de gases es una técnica única y versátil, que en su etapa inicial de desarrollo fue aplicada al análisis de gases y vapores de compuestos con mucha volatilidad. Como una herramienta analítica, puede ser utilizada para la separación directa y el análisis de muestras gaseosas, soluciones líquidas y sólidos volátiles. Puede ser aplicada a la solución de muchos problemas en varios campos como ser:

a) Drogas y Farmacéutica: En el control de calidad de productos, análisis de nuevos productos y el monitoreo de metabolitos en sistemas biológicos.

b) Estudios Medioambientales: En el campo de contaminación del aire, el análisis por Cromatografía de gases es fácilmente adoptado para la separación y análisis de diversas mezclas.

c) Industria del Petróleo: Las compañías petroleras estuvieron entre las primeras en hacer un uso generalizado de la Cromatografía de gases. La técnica fue empleada satisfactoriamente para separar y determinar los muchos componentes en los productos de petróleo.

d) Pesticidas y sus residuos: La Cromatografía de gases en combinación con detectores selectivos como el de captura de electrones (ECD), fósforo (NPD) y conductividad electrolítica, han hecho que la detección de tales compuestos y su medición sean relativamente simples.

e) Alimentos: La determinación de antioxidantes y preservantes alimenticios es una parte activa en el campo de la Cromatografía de gases.

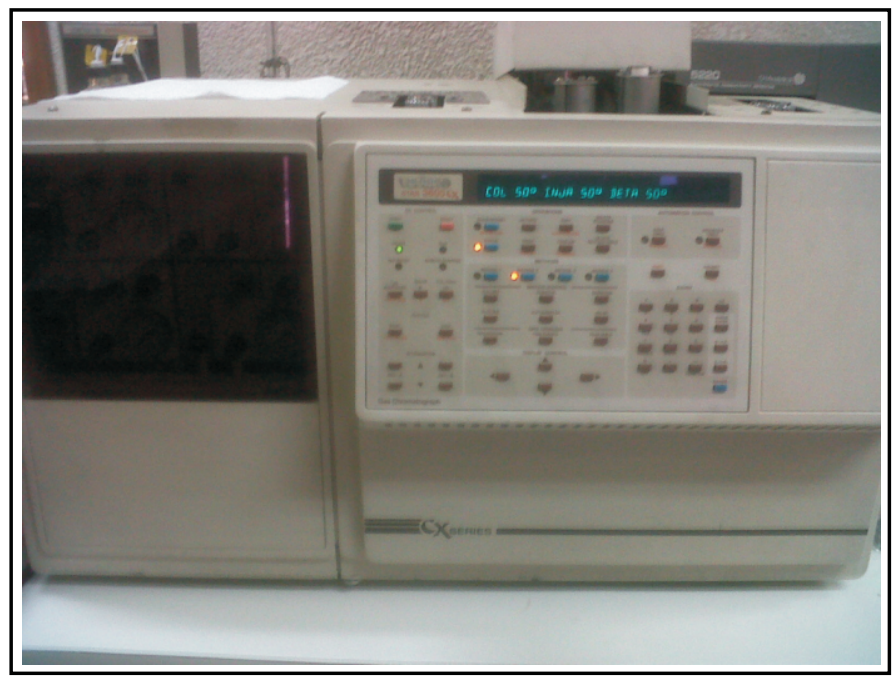

Figura 3.1. Cromatógrafo de gases Varian Cx3600. 


\section{Inyector}

Debido a que el proceso de extracción de la muestra implica la desorción de las tiras de carbono activada con el disulfuro de carbono, el método precisa de una temperatura en el puerto de inyección igual a $230 \mathrm{C}$, permitiendo de esta manera la volatilización de los componentes antes de que ingresen a la columna. Utilizando una microjeringa se inyecta un microlitro de los controles, estándares y blanco, aspirando primero un microlitro de aire, luego el microlitro de la solución a analizar y por último otro microlitro de aire.

\section{Gas acarreador}

En general la fase móvil utilizada más comúnmente son gases nobles como el Helio o Nitrógeno, utilizando en nuestro método el último por razones de incremento elevado en costos del Helio. Con el fin de evitar la detección de sustancias ajenas a la muestra (impurezas), la calidad del Nitrógeno debe ser ultrapura. La presión de salida del mismo es de 50PSI (lb/pulg), con la cual se alcanza una buena separación de los compuestos, así como alta resolución en los cromatogramas.

\section{Columna y Horno}

La columna capilar (Figura 3.2), la temperatura de la misma y la rampa de temperatura empleada son en gran medida el aspecto más importante del análisis, ya que la naturaleza del empaquetado de la columna, su afinidad por compuestos no polares y el incremento de la temperatura durante la corrida cromatográfica permiten la separación e identificación en las muestras. En el método presentado se trabaja con una temperatura inicial en la columna de 50C, manteniéndose por dos minutos, los cuales una vez transcurridos comenzará a incrementar a razón de $7 \mathrm{C}$ por minuto hasta alcanzar la temperatura final de $220 \mathrm{C}$, misma que se mantendrá (hold time) por cinco minutos, por lo que obtenemos una corrida cromatográfica de 31,28 minutos.

Originalmente se realizaron pruebas con una temperatura inicial en la columna de $60 \mathrm{C}$, manteniéndose por 2 minutos, y con una rampa de temperatura de $10 \mathrm{C}$ por minuto, con un "hold time" de 10 minutos, con lo que se obtenían cromatogramas de corridas más rápidas, pero con baja resolución, observándose asimismo que el hold time era muy elevado, ya que los compuestos de interés terminan de eluir una vez alcanzada la temperatura final. 


\section{Detector de lonización de Flama}

Desde 1958 el FID se ha convertido en el detector más ampliamente utilizado en la cromatografía de gases, principalmente porque reacciona virtualmente a todos los compuestos orgánicos con una buena sensibilidad, y la respuesta no es afectada por moderados cambios en el flujo, presión o temperatura. Además el FID no responde a las impurezas comunes del gas acarreador como el dióxido de carbono y agua en operaciones normales.

EI FID consiste en la difusión de una pequeña flama producida con hidrógeno y aire, que quema a los componentes que eluyen de la columna junto al gas acarreador y en nuestro método el disulfuro de carbono. EI FID es un detector sensitivo de masas que responde al número de átomos de carbono que ingresan por unidad de tiempo, en donde el factor de respuesta es dado por el área o la altura de pico dividida por la masa de soluto inyectado.

En general el FID es robusto, fácil de operar, con la desventaja de ser un detector destructivo de la muestra, pero que pese a ello es el detector más popular. En el método propuesto el detector FID trabaja a una temperatura de $250 \mathrm{C}$, temperatura utilizada generalmente en los métodos propuestos para la identificación de líquidos inflamables como la gasolina y diesel.

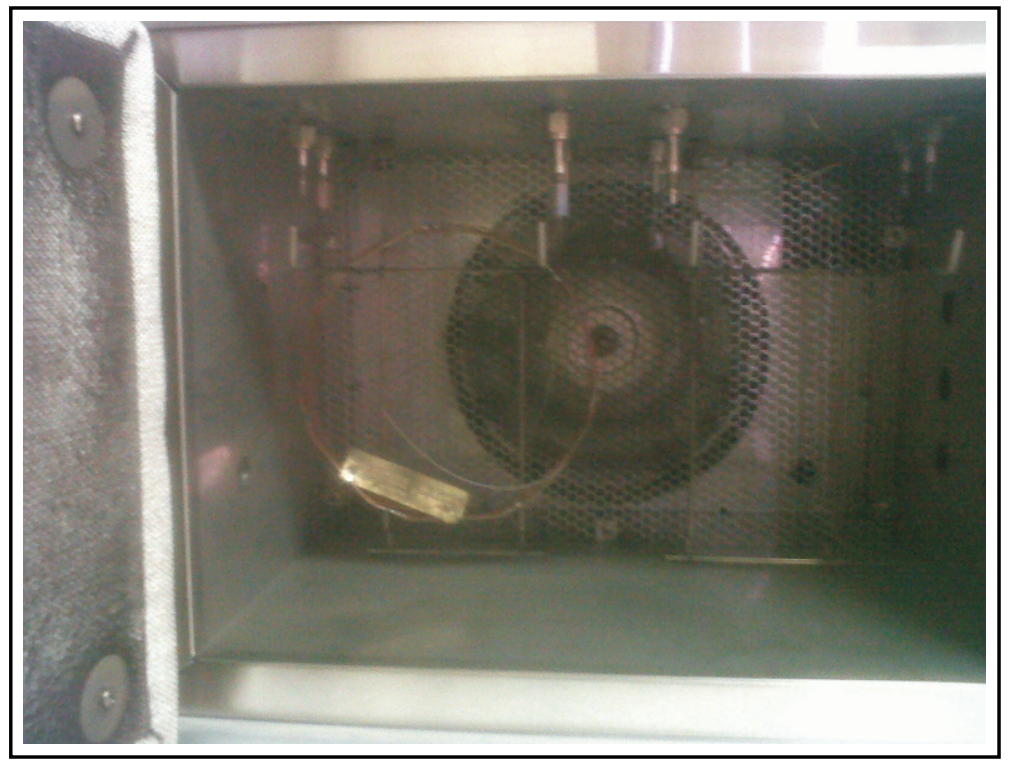

Figura 3.2. Columna DB-1 y Horno de la Columna. 


\section{CAPÍTULOIV}

\section{Resultados obtenidos}

La Cromatografía de gases es capaz de cuantificar la presencia de los analitos presentes en una mezcla determinada, sin embargo, en el caso de los líquidos inflamables en escombros de incendios, la función del laboratorio estará enfocada en señalar la presencia o no de dichas sustancias, por lo que se busca desarrollar un método analítico con un buen límite de detección, el cual dependerá en gran medida del proceso de extracción y del análisis instrumental. Es sabido que el detector FID posee una sensibilidad elevada $(\sim 10-13 \mathrm{~g} / \mathrm{s})$ con un gran intervalo de respuesta lineal ( 10 7). En el método utilizado los controles se prepararon colocando $1 \mathrm{ml}$ de productos comerciales de diesel y gasolina, respectivamente.

En el Cromatograma de la figura 4.1 se presenta la corrida del $\mathrm{CS}_{2}$, utilizado para la desorción del analito; en el mismo observamos que presenta un único pico a un tiempo de retención cercano a los 2 minutos. El Cromatograma de la figura 4.2 corresponde a la corrida del producto comercial de gasolina disuelto en $\mathrm{CS}_{2}$, viéndose los picos característicos del analito en los primeros 10015 minutos. Caso contrario ocurre con el producto comercial de diesel, el cual se presenta en el cromatograma de la figura 4.3 , donde los picos característicos comienzan a aparecer a los 8 minutos y finalizan a los 25 minutos.

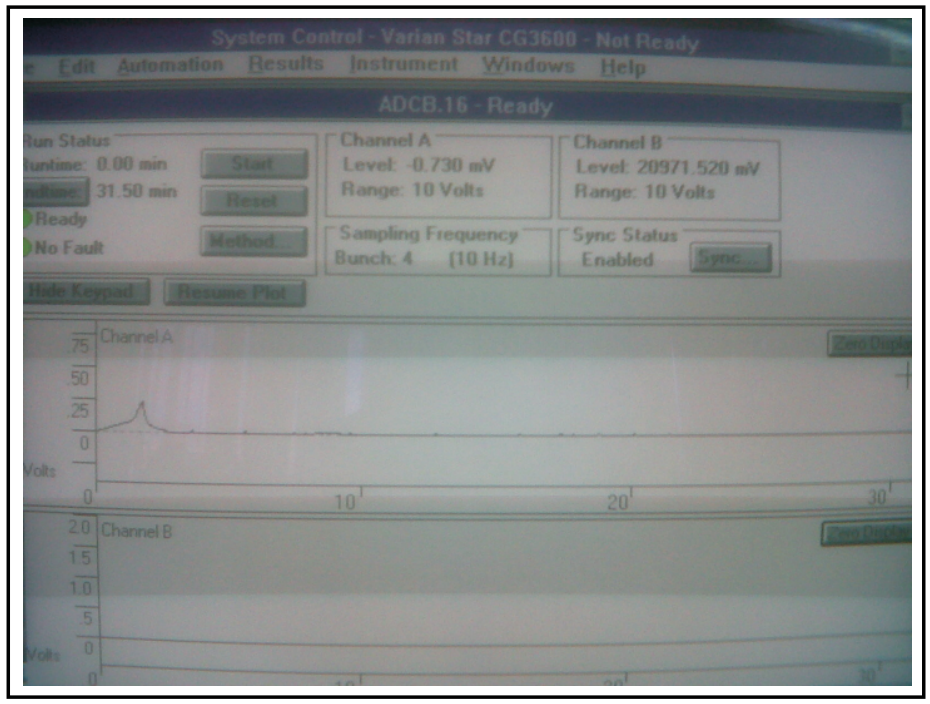

Figura 4.1. Cromatograma del $\mathrm{Cs}_{2}$ 


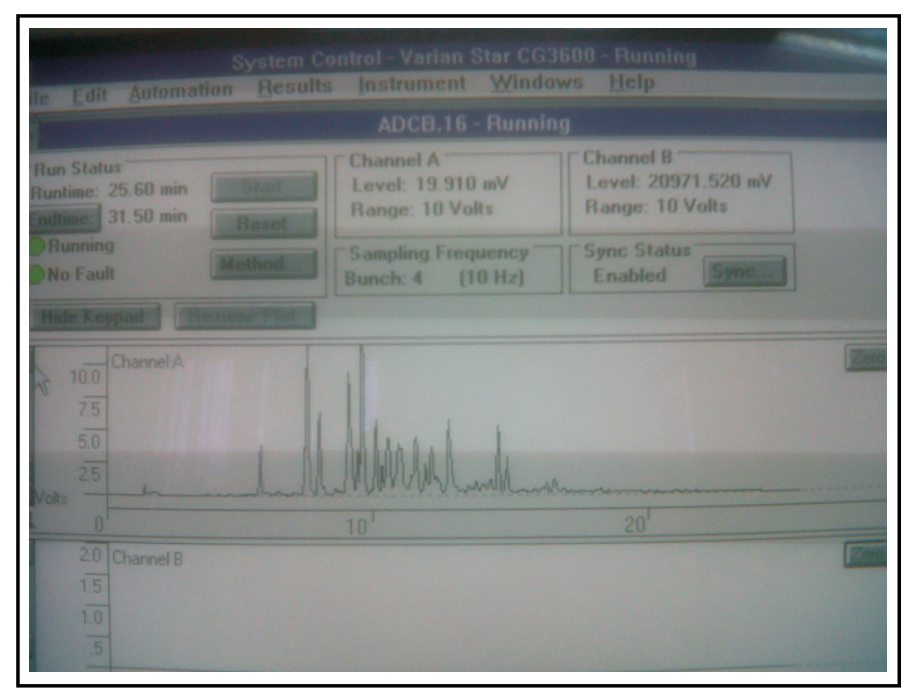

Figura 4.2. Cromatograma de producto comercial de gasolina.

Lo anterior ocurre por la volatilidad más alta de los componentes de la gasolina en comparación a los del diesel, los cuales tienen puntos de ebullición mayores. El cromatograma de la figura 4.4 nos presenta una mezcla de ambos productos comerciales en solución, en donde podemos observar la secuencia de todos los picos característicos de ambos, existiendo una zona entre los 7 y 13 minutos en que los picos son más altos por la presencia de esos compuestos en ambas mezclas.

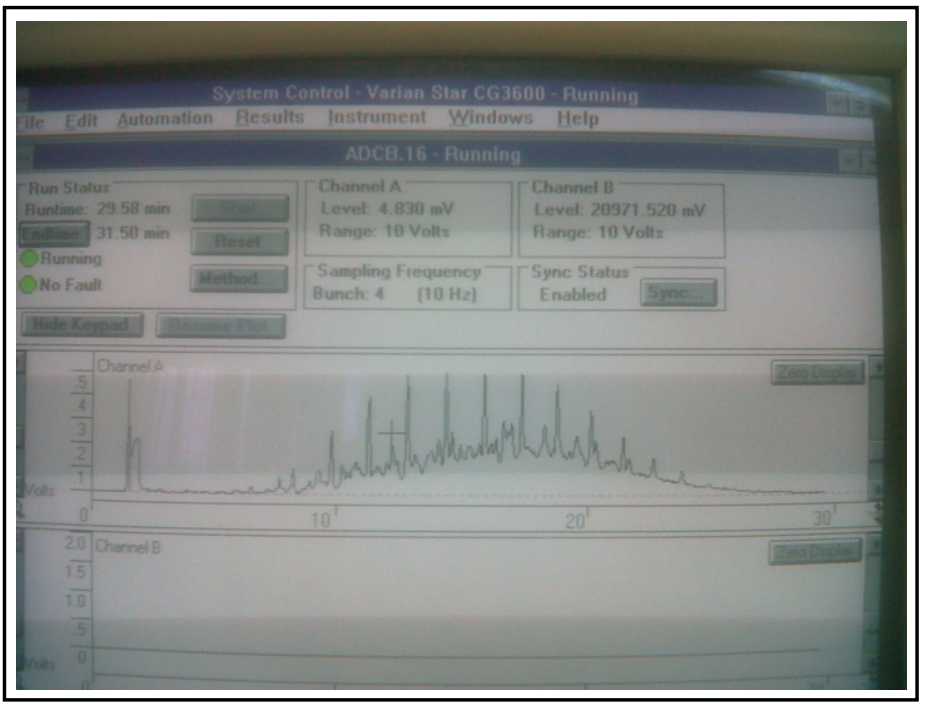

Figura 4.3. Cromatograma de producto comercial de diesel. 


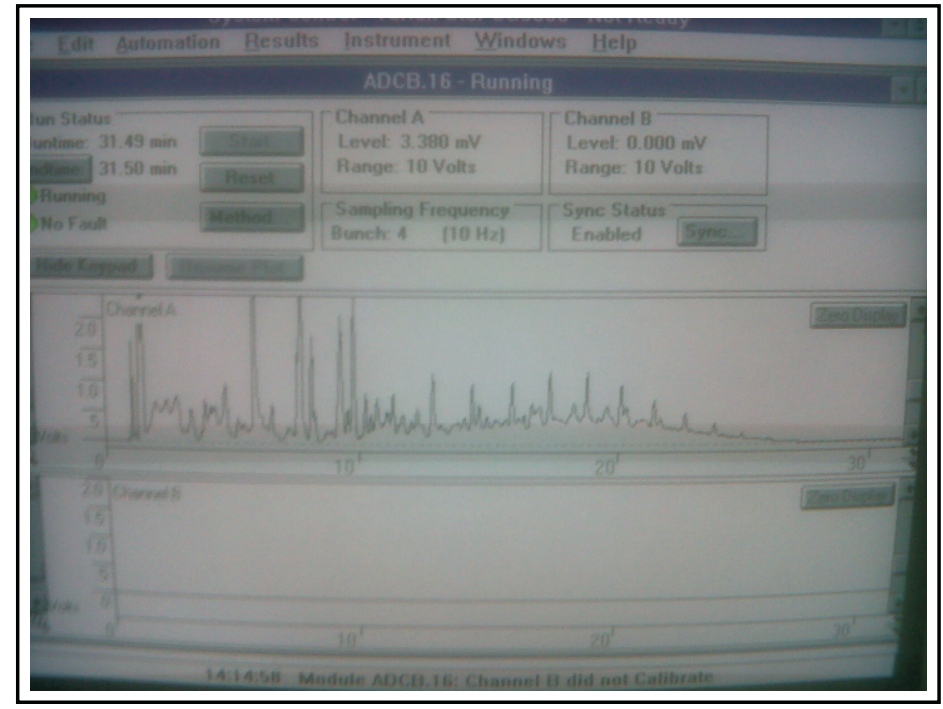

Figura 4.4. Cromatograma de mezcla de productos comerciales de gasolina y diesel.

Se presenta en el cromatograma de la figura 4.5 la corrida para el extracto del control de gasolina, posterior a su desorción con CS2, y la inyección de $1 \mathrm{mcl}$. En él podemos observar la presencia de los picos característicos, observados en el cromatograma de la solución del producto comercial. De igual forma, para el control de diesel los resultados se reflejan en el cromatograma de la figura 4.6.

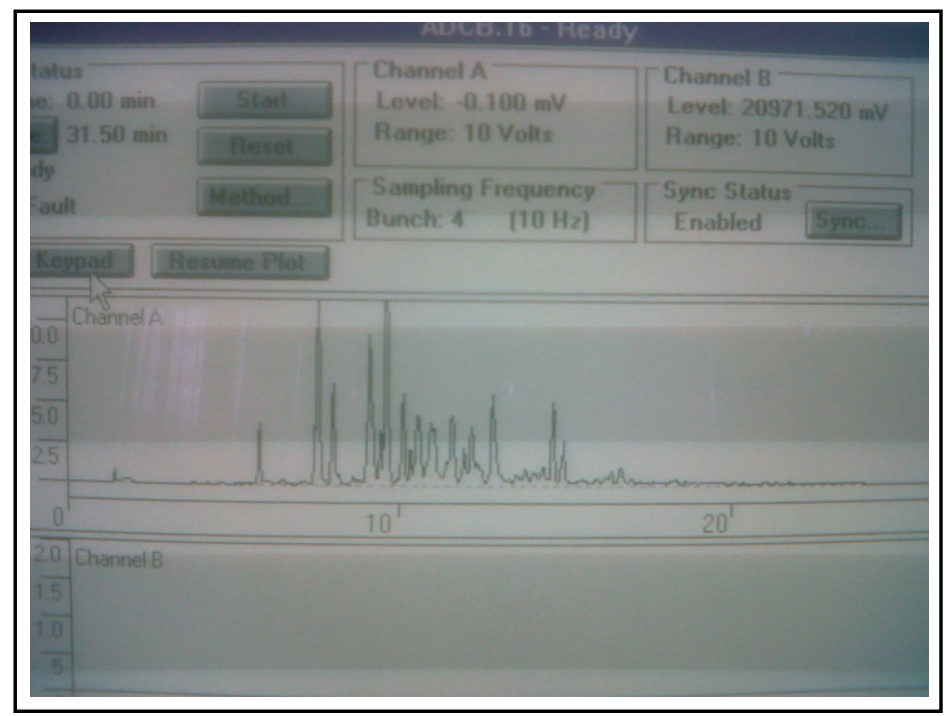

Figura 4.5. Cromatograma de control de gasolina. 


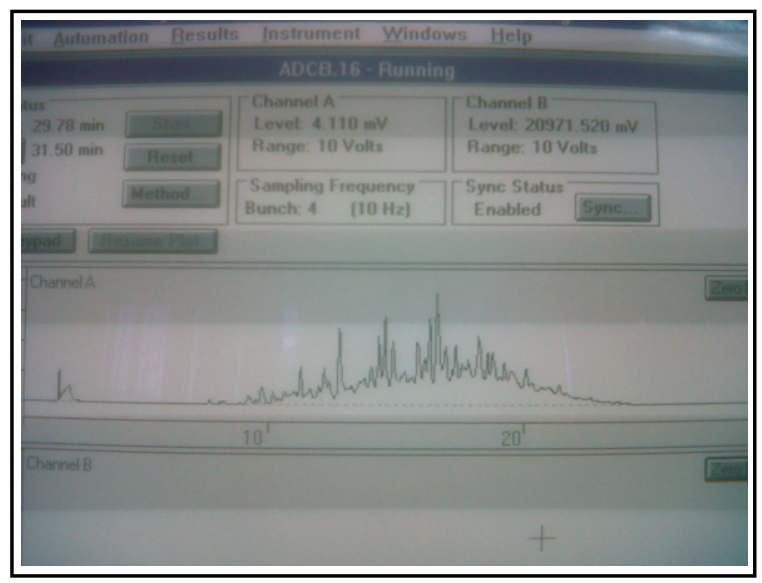

Figura 4.6. Cromatograma de control de diesel.

Desafortunadamente los datos obtenidos del análisis de los extractos de escombros no se limitan únicamente a componentes de líquidos inflamables. Algunos de los compuestos volátiles presentes en los extractos pueden incluir contaminantes de fondo, productos de pirolisis, productos de combustión incompleta y constituyente de los líquidos inflamables. Todos estos productos serán representados en los datos del cromatógrafo de gases. Esto puede resultar en patrones muy complejos en los que el analista debe discernir entre los líquidos inflamables y la contribución de la matriz. En el caso de nuestro método, el control de matriz preparado no tendrá una gran influencia de ese tipo pues no sufre ningún proceso al que sí se encontrará sometida una matriz en una investigación real. Pese a ello, el cromatograma de la figura 4.7 nos proporciona la corrida del control de matriz, en donde vemos muy pocos picos, y con poco efecto de la misma en el análisis que pueda interferir en las señales obtenidas para la gasolina y el diesel.

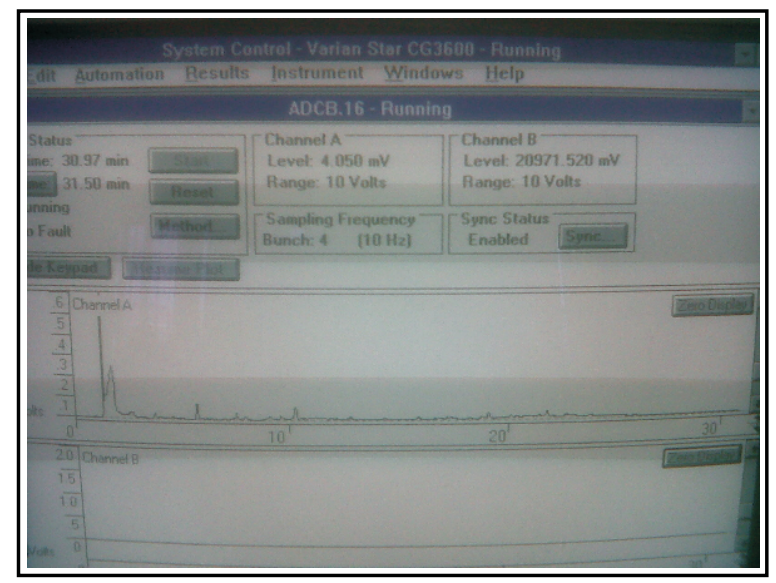

Figura 4.7. Cromatograma de control de matriz. 


\section{CONCLUSIONES}

Se logró desarrollar un método analítico para la detección de gasolina y diesel con un buen límite de detección, capaz de detectar cantidades menores a los mililitros, asimismo con las ventajas de ser rápido y confiable. El método presenta la ventaja de diferenciar la presencia de mezclas volátiles "ligeras", como la gasolina, de otras más "pesadas" como el diesel, con una corrida cromatográfica de poco más de los 30 minutos.

Siendo la etapa de preparación de la muestra de carácter importante y básico, para el buen desempeño del mismo, esto por el tipo de matriz en donde se cree se encuentran los analitos, se desarrolló el método de adsorción pasiva, capaz de extraer la suficiente cantidad para ser introducida en el cromatógrafo de gases, emitiendo éste su correspondiente señal. Se verificó la temperatura y tiempo adecuados para la adsorción pasiva en el horno, entre 80 y $90 \mathrm{C}$ durante al menos 16 horas.

Refiriéndonos a los cromatogramas obtenidos, estos poseen buena resolución, además de ser consistentes en los resultados obtenidos, tanto para los controles como para el control de matriz. Hablando de este último, se evaluó solamente la interferencia de una matriz preparada por el laboratorio, con las consideraciones de no ser una matriz real de escombros de incendios; pero como se menciona en el capítulo IV, las posibles señales encontradas en la matriz deben ser estudiadas y evaluadas por el analista, mismo que deberá hacer uso de su experiencia y conocimiento en análisis de este tipo.

El Laboratorio Químico-Toxicológico cuenta ya con un método preciso, confiable, rápido y adecuado a su propósito, el cual puede emplearse para la correcta identificación de líquidos inflamables en aquellos casos en que los investigadores sospechen de la presencia de algún líquido inflamable derivado del petróleo, especificamente, para mezclas como la gasolina y diesel. Una de las acciones a seguir es la correcta validación del método, a fin de verificar parámetros de desempeño del mismo como ser robustez, límite de cuantificación, linealidad, exactitud; entre otros parámetros recomendados por las normas internacionales.

\section{AGRADECIMIENTOS}

En los recientes años el Laboratorio Químico-Toxicológico ha tenido grandes avances, logrando ser parte fundamental en el esclarecimiento de casos de importancia para la sociedad hondureña, aplicando la prueba científica, 
acompañada la validez técnica de sus metodologías de análisis; siendo el único laboratorio de este tipo en el país, lo que le incita y forza a seguir en constante crecimiento y mejoramiento de sus técnicas y procesos. Estos avances no fueran posibles sin el valioso y decidido apoyo de parte de la escala jerárquica desde la Directora de Medicina Forense, Dra. Lucy Marder; la Asistencia de la Dirección, Dra. Mildred Alvarenga; así como la Jefatura de los Laboratorios Criminalísticos y de Ciencias Forenses, Dra. Antonieta Zúñiga. Agradezco todo el apoyo y la confianza a la Jefa del Laboratorio Químico-Toxicológico, Dra. Alba Blanco y al decano de la Facultad de Ciencias Químicas y Farmacia, Dr. René Sagastume, por ofrecerme la oportunidad de manejar el Laboratorio Instrumental de la Facultad, sitio que me ha proporcionado avances y aprendizaje en el campo del análisis químico instrumental.

\section{REFERENCIASBIBLIOGRÁFICAS}

1) R.C. Grob, e.f. Barry, L.A Colon, t.a. Brettell, I.j Baira, R.E. Lester, C.C. Walters, et al. Modern practice of gas chromatography. Cuarta edición. Estados Unidos de America. Wiley-interscience. 2004.

2) N.N. Daied, C. Maguire, R. Newman, M. Shipp, E. Satuffer, J.D. Twibell. Firel nvestigation. Estados Unidos de América. CRC Press. 2004.

3) A.D. Café. Analysis of accelerants in fire debris by capillary gas liquid chromatography. Australia. University of Technology Sydney. 1988.

4) Basic tools and resources for fire investigators: a handbook. Estados Unidos de América. Federal emergency management agency. 1992.

5) P.C. Sadek. Illustrated pocket dictionary of chromatography. Estados Unidos de América. Wiley-Interscience. 2004.

6) D.A. Skoog, f.j. holler, t.a. Nieman. Principios de análisis instrumental. Quinta edición. España. Mcgraw hill. 2001.

7) P.M. I. Sandercock. "Fire investigation and ignitable liquid residue analysis- a review: 2001-207" Forensic Science International. 176. 2008. 93-110.

8) M. Frenkel, S. Tsaroom, Z. Aizenshtat, S. Kraus, D. Daphna. "Enhanced sensitivity in analysis of arson residues: an adsorption-tube/gas chromatography method" Journal of Forensic Sciences, jfsca. 29. 3. 1984. 723-731.

9) M.R. Williams, D. Fernandes, C. Bridge, D. Darrien, S. Elliott, M. Sigman. "Adsorption saturation and chromatography distortion effects on passive headspace sampling with activated charcoal in fire debris analysis" J. Forensic sci. 50.2.2005.

10)S. Kurata, T. lyuzumi, H. Hirano, M. Nagai. "Discrimination between residues from kerosene or gas oil and plastics in fire debris" Journal of the Japan Petroleum Institute. 50. 2. 2007.69-78. 
11) S. Casamento, E. Kunza, E. Du Pasquier, D. Royas. "Evaluation of a portable gas crhromatography for the detection of ignitable liquids" Can. Soc. Forensic sci. j. 38. 4.2005.191-203.

12) Standard test methods for ignitable liquid residues in extracts from fire debris samples by gas chromatography. astm e1387-01.

13) Standard practice for separation of ignitable liquid residues from fire debris samples by passive headspace concentration whit activated charcoal. astm e1412-00. 